CRYSTALLOGRAPHIC COMMUNICATIONS
Received 2 February 2017

Accepted 23 March 2017

Edited by H. Ishida, Okayama University, Japan

Keywords: crystal structure; nickel(II) complex; 2-aminobenzaldehyde; asymmetric structure; supramolecular structure.

CCDC reference: 1539785

Supporting information: this article has supporting information at journals.iucr.org/e

\section{Crystal structure of [2-(\{2-[(2-azanidylbenzylidene)- amino]benzylidene\}amino)-4-chlorophenolato]- nickel(II)}

Fumiya Kobayashi, Atsushi Koga, Ryo Ohtani, Shinya Hayami and Masaaki

Nakamura*

Department of Chemistry, Graduate School of Science and Technology, Kumamoto University, 2-39-1 Kurokami, Kumamoto 860-8555, Japan. *Correspondence e-mail: nakamura@sci.kumamoto-u.ac.jp

The title complex, $\left[\mathrm{Ni}\left(\mathrm{C}_{20} \mathrm{H}_{14} \mathrm{ClN}_{3} \mathrm{O}\right)\right]$, with an asymmetrically chlorideappended Schiff base ligand has been synthesized and structurally characterized at $100 \mathrm{~K}$. In the compound, the central nickel(II) ion has a square-planar coordination geometry with $\mathrm{N}_{3} \mathrm{O}$ donors of the $\pi$-conjugated tetradentate Schiff base ligand. In the crystal, the complexes are connected into an inversion dimer via an $\mathrm{Ni} \cdot \mathrm{Ni}$ interaction $[3.1753(5) \AA]$ and a pair of $\pi-\pi$ interactions [centroid-centroid distance $=3.8416(16) \AA]$. The dimers are linked via a $\mathrm{C}-\mathrm{H} \cdots \mathrm{Cl}$ hydrogen bond, forming a chain along the $c$-axis direction. The dimer chains interact with each other through $\pi-\pi$ interactions [centroid-centroid distance $=3.8736(16) \AA$ ], forming a layer expanding parallel to the $a c$ plane.

\section{Chemical context}

Metal complexes with a tetradentate Schiff base ligand as represented by $\mathrm{H}_{2}$ (salen) [N,N'-ethylenebis(salicylideneimine)] and its derivatives have played extremely important roles in the field of coordination chemistry. Up to now, a large number of salen derivatives have been prepared and used for complexation in the expectation of a wide range of features such as catalytic ability, magnetic, dielectric and luminescence properties and so on (Bermejo et al., 1996). In these cases, symmetric tetradentate ligands mainly produce $\mathrm{N}_{2} \mathrm{O}_{2}$ or $\mathrm{N}_{4}$ type coordination environments. In this research, we have designed asymmetric structures, both in the coordination environment and in the molecular configuration, for the construction of the supramolecular structure through intermolecular hydrogen bonds, and synthesized the title nickel(II) complex using an asymmetrically chloride-appended tetradentate Schiff base ligand.

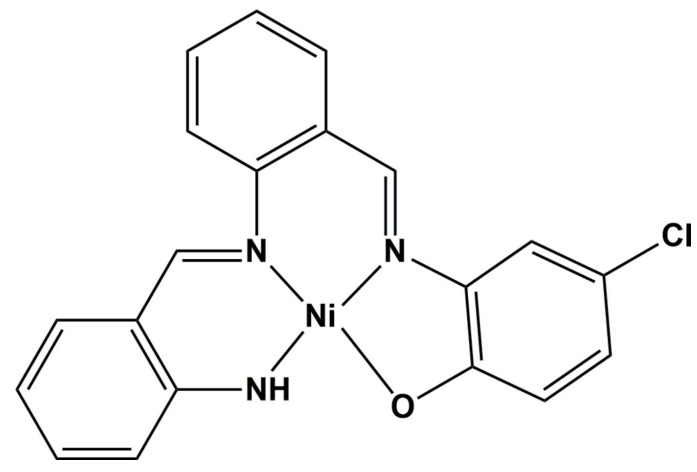




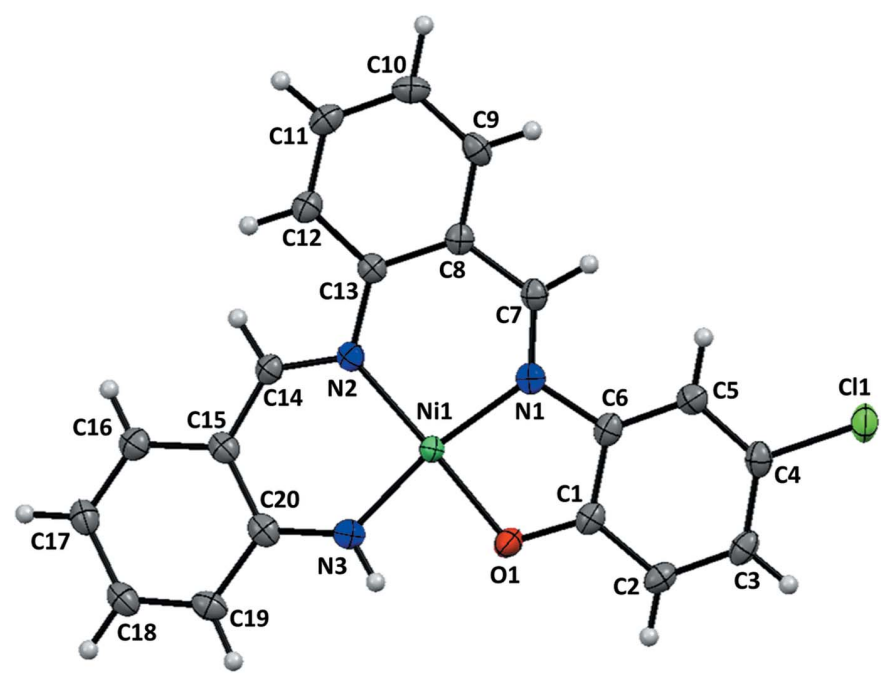

Figure 1

The molecular structure of the title compound, showing displacement ellipsoids at the $50 \%$ probability level.

The structure of the title compound, which features a widely spread $\pi$-conjugated ring system, is also useful for supramolecular assemblies through $\pi-\pi$ interactions. The mononuclear copper(II) complex with a similar $\mathrm{N}_{3} \mathrm{O}$ type asymmetrical ligand was reported by Ghorai \& Mukherjee (2014).

\section{Structural commentary}

The nickel(II) atom is in a square-planar coordination with an asymmetrical coordination environment formed by the $\mathrm{N}_{3} \mathrm{O}$

(a)

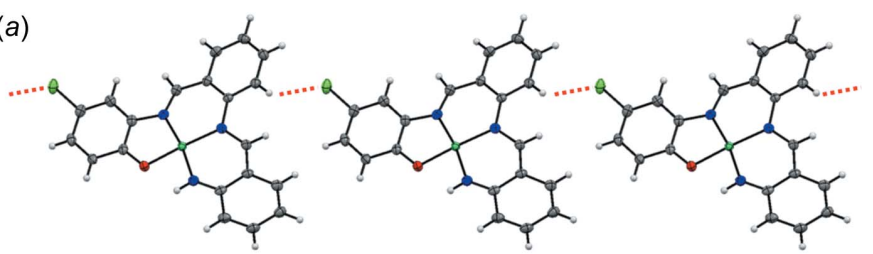

(b)

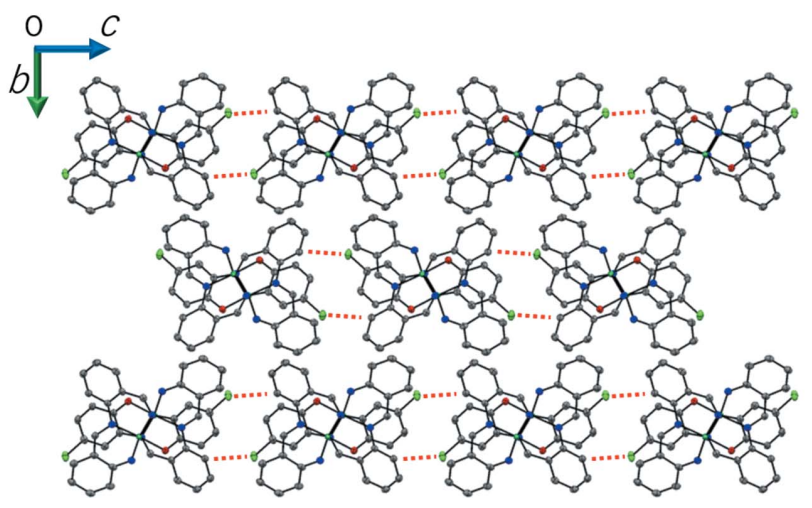

Figure 2

Packing diagrams of the title compound, showing $(a)$ a chain structure running along the $c$ axis formed by $\mathrm{C}-\mathrm{H} \cdots \mathrm{Cl}$ hydrogen bonds (red dashed lines) and ( $b$ ) the chains viewed along the $a$ axis.
Table 1

Hydrogen-bond geometry $\left(\AA{ }^{\circ}\right)$.

\begin{tabular}{lllll}
\hline$D-\mathrm{H} \cdots A$ & $D-\mathrm{H}$ & $\mathrm{H} \cdots A$ & $D \cdots A$ & $D-\mathrm{H} \cdots A$ \\
\hline $\mathrm{C} 12-\mathrm{H} 12 \cdots \mathrm{C} 11^{\mathrm{i}}$ & 0.95 & 2.76 & $3.540(3)$ & 140 \\
$\mathrm{C} 10-\mathrm{H} 10 \cdots \mathrm{C}^{\mathrm{ii}}{ }^{\mathrm{i}}$ & 0.95 & 2.80 & $3.626(4)$ & 146 \\
\hline
\end{tabular}

Symmetry codes: (i) $x, y, z-1$; (ii) $-x, y-\frac{1}{2},-z+\frac{1}{2}$.

donor set including one phenolate $\mathrm{O}$ atom, two imine $\mathrm{N}$ atoms and one amino $\mathrm{N}$ atom of the tetradentate Schiff base ligand (Fig. 1). The $\mathrm{Ni}-\mathrm{O} 1, \mathrm{Ni}-\mathrm{N} 1, \mathrm{Ni}-\mathrm{N} 2$, and $\mathrm{Ni}-\mathrm{N} 3$ bond lengths are 1.8617 (18), 1.878 (2), 1.896 (2) and 1.831 (2) $\AA$, respectively. The complex molecule is approximately planar; the coordination plane $(\mathrm{N} 1-\mathrm{N} 3 / \mathrm{O} 1 / \mathrm{Ni1})$ makes dihedral angles of $4.15(12), 10.22(12)$ and $13.42(12)^{\circ}$, respectively, with the $\mathrm{C} 1-\mathrm{C} 6, \mathrm{C} 8-\mathrm{C} 13$ and $\mathrm{C} 15-\mathrm{C} 20$ benzene rings.

\section{Supramolecular features}

In the crystal, pairs of complex molecules related by an inversion centre are dimerized by an $\mathrm{Ni} \cdot \mathrm{Ni}$ interaction [3.1753 (5) $\AA$ ] and a pair of $\pi-\pi$ interactions between the $\mathrm{C} 1-$ $\mathrm{C} 6$ and $\mathrm{C} 15-\mathrm{C} 20$ benzene rings [centroid-centroid distance $=$ $3.8415(16) \AA]$. Such dimerization caused by an $\mathrm{Ni} \cdots \mathrm{Ni}$ interaction has also been observed in symmetric $\mathrm{Ni}$ (salen) compounds (Aullón et al., 1996; Siegler \& Lutz, 2009). The dimeric molecules of the title compound are linked by $\mathrm{C}-$ $\mathrm{H}$. C Cl hydrogen bonds (Table 1), producing a chain of dimers along the $c$ axis (Fig. 2). The dimers further interact with each other through $\pi-\pi$ interactions between the $\mathrm{C} 1-\mathrm{C} 6$ and $\mathrm{C} 8-$ $\mathrm{C} 13$ benzene rings [centroid-centroid distance = 3.8738 (17) $\AA$ ], forming a column along the $a$ axis (Fig. 3). Together, these $\mathrm{C}-\mathrm{H} \cdots \mathrm{Cl}$ and $\pi-\pi$ interactions result in a layer parallel to the $a c$ plane. The layers are further linked by a short $\mathrm{C}-\mathrm{H} \cdots \mathrm{C}$ contact (Table 1 ), giving a three-dimensional network (Fig. 4).

\section{Synthesis and crystallization}

The tetradentate Schiff base ligand was prepared by the reaction of 2-aminobenzaldehyde (Smith \& Opie, 1948) $(0.228 \mathrm{~g}, 2.0 \mathrm{mmol})$ and 2-amino-4-chlorophenol (0.144 g,

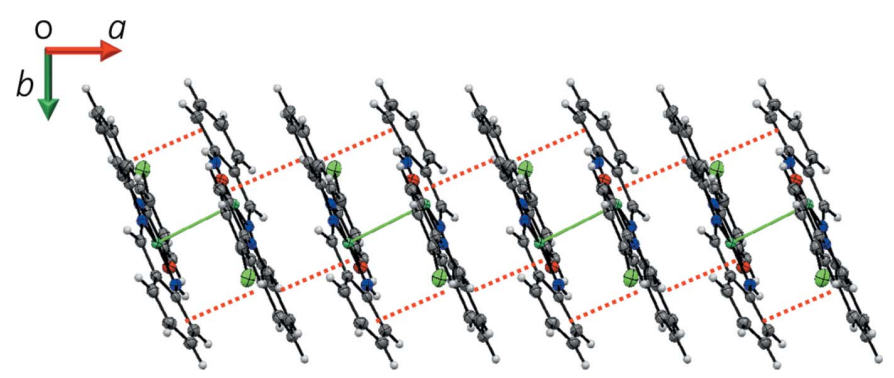

Figure 3

A packing diagram of the title compound, showing the column structure along the $a$ axis formed by $\mathrm{Ni} \cdots \mathrm{Ni}$ interactions (green solid lines) and $\pi$ $\pi$ interactions (red dashed lines). 
(a)

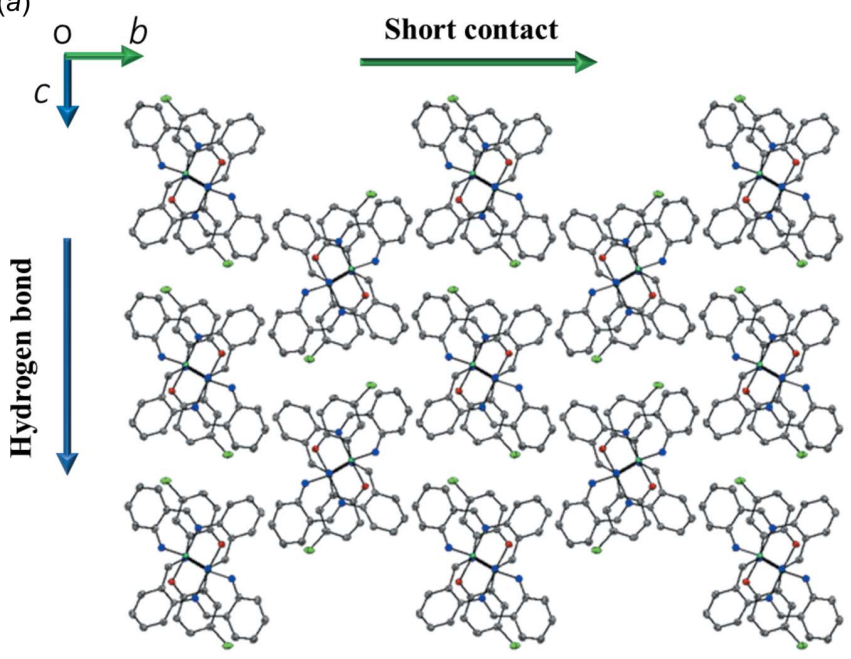

(b)

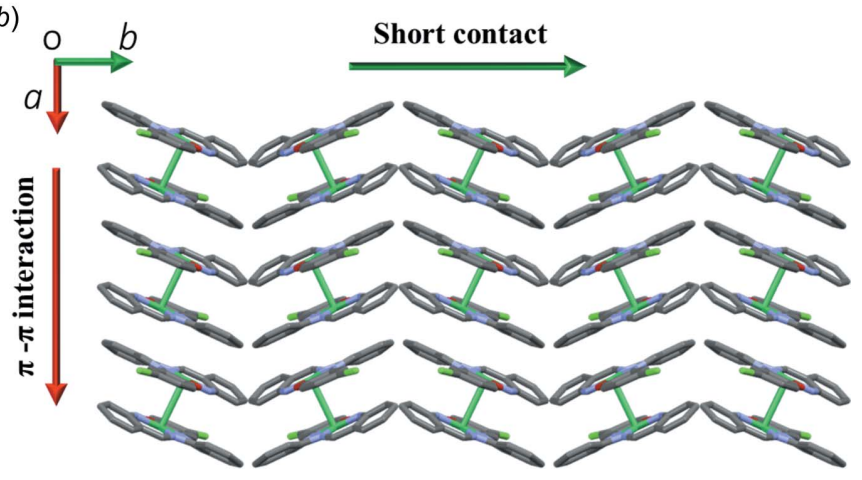

Figure 4

Packing diagrams of the title compound assembled by $($ a) $\mathrm{C}-\mathrm{H} \cdots \mathrm{Cl}$ hydrogen bonds and $\mathrm{C}-\mathrm{H} \cdots \mathrm{C}$ short contacts, and $(b) \pi-\pi$ interactions and short contacts.

$1.0 \mathrm{mmol})$ in methanol $(50 \mathrm{ml})$ under stirring for $1 \mathrm{~h}$. The resulting solution including the ligand was used for complexation with the $\mathrm{Ni}^{\mathrm{II}}$ ion. A methanol solution $(50 \mathrm{ml})$ of $\mathrm{Ni}\left(\mathrm{CH}_{3} \mathrm{COO}\right)_{2} \cdot 4 \mathrm{H}_{2} \mathrm{O}(0.249 \mathrm{~g}, 1.0 \mathrm{mmol})$ was added to the solution and stirred for $1 \mathrm{~h}$. The resulting solution was allowed to stand for a few days, during which time dark-purple blockshaped crystals precipitated. They were collected by suction filtration and dried in air to give single crystals of the title compound suitable for X-ray diffraction.

\section{Refinement}

Crystal data, data collection and structure refinement details are summarized in Table 2. The position of the $\mathrm{N}$-bound $\mathrm{H}$ atom was refined with $\mathrm{N}-\mathrm{H}=0.86(1) \AA$ and $U_{\text {iso }}(\mathrm{H})=$ $1.5 U_{\text {eq }}(\mathrm{N})$. Other $\mathrm{H}$ atoms were treated as riding with $\mathrm{C}-\mathrm{H}=$ $0.95 \AA$ and $U_{\text {iso }}(\mathrm{H})=1.2 U_{\text {eq }}(\mathrm{C})$.
Table 2

Experimental details.

\begin{tabular}{|c|c|}
\hline \multicolumn{2}{|l|}{ Crystal data } \\
\hline Chemical formula & {$\left[\mathrm{Ni}\left(\mathrm{C}_{20} \mathrm{H}_{14} \mathrm{ClN}_{3} \mathrm{O}\right)\right]$} \\
\hline$M_{\mathrm{r}}$ & 406.50 \\
\hline Crystal system, space group & Monoclinic, $P 2_{1} / c$ \\
\hline Temperature (K) & 100 \\
\hline$a, b, c(\AA)$ & $7.5510(4), 17.8689(9), 12.6834(6)$ \\
\hline$\beta\left(^{\circ}\right)$ & $109.9504(14)$ \\
\hline$V\left(\AA^{6}\right)$ & $1608.64(14)$ \\
\hline$Z$ & 4 \\
\hline Radiation type & Мo $K \alpha$ \\
\hline$\mu\left(\mathrm{mm}^{-1}\right)$ & 1.39 \\
\hline Crystal size $(\mathrm{mm})$ & $0.46 \times 0.27 \times 0.25$ \\
\hline \multicolumn{2}{|l|}{ Data collection } \\
\hline Diffractometer & Rigaku R-AXIS RAPID \\
\hline Absorption correction & $\begin{array}{l}\text { Multi-scan (ABSCOR; Higashi, } \\
\text { 1995) }\end{array}$ \\
\hline$T_{\min }, T_{\max }$ & $0.476,0.712$ \\
\hline $\begin{array}{l}\text { No. of measured, independent and } \\
\text { observed }\left[F^{2}>2.0 \sigma\left(F^{2}\right)\right] \text { reflec- } \\
\text { tions }\end{array}$ & $15243,3638,3177$ \\
\hline$R_{\mathrm{int}}$ & 0.039 \\
\hline$(\sin \theta / \lambda)_{\max }\left(\AA^{-1}\right)$ & 0.647 \\
\hline \multicolumn{2}{|l|}{ Refinement } \\
\hline$R\left[F^{2}>2 \sigma\left(F^{2}\right)\right], w R\left(F^{2}\right), S$ & $0.042,0.103,1.04$ \\
\hline No. of reflections & 3638 \\
\hline No. of parameters & 238 \\
\hline No. of restraints & 1 \\
\hline $\mathrm{H}$-atom treatment & $\begin{array}{l}\mathrm{H} \text { atoms treated by a mixture of } \\
\text { independent and constrained } \\
\text { refinement }\end{array}$ \\
\hline$\Delta \rho_{\max }, \Delta \rho_{\min }\left(\mathrm{e} \AA^{-3}\right)$ & $1.35,-0.37$ \\
\hline
\end{tabular}

Computer programs: RAPID-AUTO (Rigaku, 1995), SHELXS2013 (Sheldrick, 2008), SHELXL2016 (Sheldrick, 2015) and CrystalStructure (Rigaku, 2014).

\section{Funding information}

Funding for this research was provided by: JSPS Grant-in-Aid for Young Scientists (B) (award No. JP15K17833); JSPS Grant-in-Aid for Scientific Research on Innovative Areas (Dynamical Ordering and Integrated Functions) (award No. JP16H00777); KAKENHI Grant-in-Aid for Scientific Research (B) (award No. JP26288026); Cooperative Research Program of the Network Joint Research Centre for Materials and Devices.

\section{References}

Aullón, G., Alemany, P. \& Alvarez, S. (1996). Inorg. Chem. 35, 50615067.

Bermejo, M. R., Castiñeiras, A., Garcia-Monteagudo, J. C., Rey, M., Sousa, A., Watkinson, M., McAuliffe, C. A., Pritchard, R. G. \& Beddoes, R. L. (1996). J. Chem. Soc. Dalton Trans. pp. 2935-2944. Ghorai, S. \& Mukherjee, C. (2014). Chem. Asian J. 9, 3518-3524.

Higashi, T. (1995). ABSCOR. Rigaku Corporation, Tokyo, Japan.

Rigaku (1995). RAPID-AUTO. Rigaku Corporation, Tokyo, Japan.

Rigaku (2014). CrystalStructure. Rigaku Corporation, Tokyo, Japan. Sheldrick, G. M. (2008). Acta Cryst. A64, 112-122.

Sheldrick, G. M. (2015). Acta Cryst. C71, 3-8.

Siegler, M. A. \& Lutz, M. (2009). Cryst. Growth Des. 9, 1194-1200. Smith, L. I. \& Opie, J. W. (1948). Org. Synth. 28, 11. 


\section{supporting information}

Acta Cryst. (2017). E73, 637-639 [https://doi.org/10.1107/S2056989017004613]

Crystal structure of [2-(\{2-[(2-azanidylbenzylidene)amino]benzylidene\}amino)-4-chlorophenolato]nickel(II)

Fumiya Kobayashi, Atsushi Koga, Ryo Ohtani, Shinya Hayami and Masaaki Nakamura

Computing details

Data collection: RAPID-AUTO (Rigaku, 1995); cell refinement: RAPID-AUTO (Rigaku, 1995); data reduction: RAPIDAUTO (Rigaku, 1995); program(s) used to solve structure: SHELXS2013 (Sheldrick, 2008); program(s) used to refine structure: SHELXL2016 (Sheldrick, 2015); molecular graphics: CrystalStructure (Rigaku, 2014); software used to prepare material for publication: CrystalStructure (Rigaku, 2014).

[2-(\{2-[(2-Azanidylbenzylidene)amino]benzylidene\}amino)-4-chlorophenolato]nickel(II)

Crystal data

$\left[\mathrm{Ni}\left(\mathrm{C}_{20} \mathrm{H}_{14} \mathrm{ClN}_{3} \mathrm{O}\right)\right]$

$M_{r}=406.50$

Monoclinic, $P 2{ }_{1} / c$

$a=7.5510(4) \AA$

$b=17.8689(9) \AA$

$c=12.6834(6) \AA$

$\beta=109.9504(14)^{\circ}$

$V=1608.64(14) \AA^{3}$

$Z=4$

\section{Data collection}

Rigaku R-AXIS RAPID diffractometer

Detector resolution: 10.000 pixels $\mathrm{mm}^{-1}$ $\omega$ scans

Absorption correction: multi-scan (ABSCOR; Higashi, 1995)

$T_{\text {min }}=0.476, T_{\max }=0.712$

15243 measured reflections

\section{Refinement}

Refinement on $F^{2}$

$R\left[F^{2}>2 \sigma\left(F^{2}\right)\right]=0.042$

$w R\left(F^{2}\right)=0.103$

$S=1.04$

3638 reflections

238 parameters

1 restraint

Primary atom site location: structure-invariant direct methods
$F(000)=832.00$

$D_{\mathrm{x}}=1.678 \mathrm{Mg} \mathrm{m}^{-3}$

Mo $K \alpha$ radiation, $\lambda=0.71075 \AA$

Cell parameters from 13442 reflections

$\theta=3.0-27.4^{\circ}$

$\mu=1.39 \mathrm{~mm}^{-1}$

$T=100 \mathrm{~K}$

Block, purple

$0.46 \times 0.27 \times 0.25 \mathrm{~mm}$

3638 independent reflections

3177 reflections with $F^{2}>2.0 \sigma\left(F^{2}\right)$

$R_{\text {int }}=0.039$

$\theta_{\max }=27.4^{\circ}, \theta_{\min }=3.0^{\circ}$

$h=-9 \rightarrow 8$

$k=-23 \rightarrow 23$

$l=-16 \rightarrow 16$

Secondary atom site location: difference Fourier map

Hydrogen site location: inferred from neighbouring sites

$\mathrm{H}$ atoms treated by a mixture of independent and constrained refinement

$w=1 /\left[\sigma^{2}\left(F_{\mathrm{o}}{ }^{2}\right)+(0.0527 P)^{2}+1.948 P\right]$

where $P=\left(F_{\mathrm{o}}{ }^{2}+2 F_{\mathrm{c}}{ }^{2}\right) / 3$

$(\Delta / \sigma)_{\max }=0.001$

$\Delta \rho_{\max }=1.35$ e $\AA^{-3}$

$\Delta \rho_{\min }=-0.37$ e $\AA^{-3}$ 


\section{Special details}

Geometry. All esds (except the esd in the dihedral angle between two 1.s. planes) are estimated using the full covariance matrix. The cell esds are taken into account individually in the estimation of esds in distances, angles and torsion angles; correlations between esds in cell parameters are only used when they are defined by crystal symmetry. An approximate (isotropic) treatment of cell esds is used for estimating esds involving l.s. planes.

Refinement. Refinement was performed using all reflections. The weighted R-factor (wR) and goodness of fit (S) are based on $\mathrm{F}^{2}$. R-factor (gt) are based on $\mathrm{F}$. The threshold expression of $\mathrm{F}^{2}>2.0 \operatorname{sigma}\left(\mathrm{F}^{2}\right)$ is used only for calculating Rfactor (gt).

Fractional atomic coordinates and isotropic or equivalent isotropic displacement parameters $\left(\AA^{2}\right)$

\begin{tabular}{|c|c|c|c|c|}
\hline & $x$ & $y$ & $z$ & $U_{\text {iso }} * / U_{\text {eq }}$ \\
\hline Ni1 & $0.29838(4)$ & $0.53846(2)$ & $0.46051(3)$ & $0.01597(11)$ \\
\hline $\mathrm{Cl1}$ & $0.22371(10)$ & $0.39200(4)$ & $0.93185(6)$ & $0.03146(17)$ \\
\hline $\mathrm{O} 1$ & $0.3645(3)$ & $0.58686(10)$ & $0.59854(15)$ & $0.0230(4)$ \\
\hline N1 & $0.2079(3)$ & $0.46083(11)$ & $0.52811(18)$ & $0.0195(4)$ \\
\hline $\mathrm{N} 2$ & $0.2225(3)$ & $0.49436(12)$ & $0.31594(18)$ & $0.0193(4)$ \\
\hline N3 & $0.3976(3)$ & $0.62220(12)$ & 0.41867 (19) & $0.0221(4)$ \\
\hline $\mathrm{C} 1$ & $0.3301(4)$ & $0.54570(14)$ & $0.6762(2)$ & $0.0205(5)$ \\
\hline $\mathrm{C} 2$ & $0.3738(4)$ & $0.56917(15)$ & $0.7885(2)$ & $0.0229(5)$ \\
\hline $\mathrm{C} 3$ & $0.3380(4)$ & $0.52243(15)$ & $0.8656(2)$ & $0.0237(5)$ \\
\hline $\mathrm{C} 4$ & $0.2594(4)$ & $0.45198(15)$ & $0.8321(2)$ & $0.0236(5)$ \\
\hline $\mathrm{C} 5$ & $0.2109(4)$ & $0.42792(15)$ & $0.7223(2)$ & $0.0224(5)$ \\
\hline C6 & $0.2447(4)$ & $0.47561(15)$ & $0.6445(2)$ & $0.0207(5)$ \\
\hline $\mathrm{C} 7$ & 0.1169 (4) & $0.40146(14)$ & $0.4813(2)$ & $0.0210(5)$ \\
\hline $\mathrm{C} 8$ & $0.0936(3)$ & $0.37840(14)$ & 0.3689 (2) & $0.0197(5)$ \\
\hline C9 & $0.0092(4)$ & $0.30739(15)$ & $0.3366(2)$ & $0.0235(5)$ \\
\hline $\mathrm{C} 10$ & $-0.0089(4)$ & $0.27609(15)$ & $0.2338(2)$ & $0.0259(6)$ \\
\hline C11 & 0.0649 (4) & $0.31435(15)$ & $0.1634(2)$ & $0.0251(5)$ \\
\hline $\mathrm{C} 12$ & $0.1469(4)$ & $0.38395(15)$ & $0.1918(2)$ & $0.0230(5)$ \\
\hline $\mathrm{C} 13$ & $0.1542(3)$ & $0.41975(13)$ & $0.2923(2)$ & $0.0187(5)$ \\
\hline $\mathrm{C} 14$ & $0.2147(4)$ & $0.53457(14)$ & $0.2259(2)$ & $0.0199(5)$ \\
\hline $\mathrm{C} 15$ & $0.2954(4)$ & $0.60452(14)$ & $0.2207(2)$ & $0.0214(5)$ \\
\hline C16 & $0.2781(4)$ & $0.63380(15)$ & $0.1133(2)$ & $0.0247(5)$ \\
\hline $\mathrm{C} 17$ & $0.3660(4)$ & $0.69883(15)$ & $0.1023(2)$ & $0.0262(5)$ \\
\hline $\mathrm{C} 18$ & $0.4758(4)$ & $0.73809(15)$ & $0.2007(2)$ & $0.0267(6)$ \\
\hline C19 & $0.4922(4)$ & $0.71300(14)$ & $0.3054(2)$ & $0.0253(6)$ \\
\hline $\mathrm{C} 20$ & $0.3979(3)$ & $0.64586(14)$ & $0.3194(2)$ & $0.0210(5)$ \\
\hline H1 & $0.452(4)$ & $0.6459(17)$ & $0.478(2)$ & $0.0315^{*}$ \\
\hline $\mathrm{H} 2$ & 0.42785 & 0.61711 & 0.81103 & $0.0275^{*}$ \\
\hline H3 & 0.36681 & 0.5382 & 0.94116 & $0.0285^{*}$ \\
\hline H5 & 0.15584 & 0.38005 & 0.70055 & $0.0268^{*}$ \\
\hline H7 & 0.06228 & 0.37131 & 0.52382 & $0.0253^{*}$ \\
\hline H9 & -0.03614 & 0.28057 & 0.38682 & $0.0282^{*}$ \\
\hline H10 & -0.07053 & 0.22937 & 0.21193 & $0.0310^{*}$ \\
\hline H11 & 0.05913 & 0.2923 & 0.09418 & $0.0301^{*}$ \\
\hline H12 & 0.19936 & 0.40819 & 0.14264 & $0.0276^{*}$ \\
\hline H14 & 0.14485 & 0.51283 & 0.15561 & $0.0239 *$ \\
\hline
\end{tabular}




\begin{tabular}{lllll} 
H16 & 0.2041 & 0.60767 & 0.04782 & $0.0296^{*}$ \\
H17 & 0.35399 & 0.71755 & 0.03007 & $0.0314^{*}$ \\
H18 & 0.53905 & 0.7827 & 0.19338 & $0.0321^{*}$ \\
H19 & 0.56662 & 0.74021 & 0.36959 & $0.0304^{*}$ \\
\hline
\end{tabular}

Atomic displacement parameters $\left(\AA^{2}\right)$

\begin{tabular}{lllllll}
\hline & $U^{11}$ & $U^{22}$ & $U^{33}$ & $U^{12}$ & $U^{13}$ & $U^{23}$ \\
\hline Ni1 & $0.01684(17)$ & $0.01610(17)$ & $0.01561(17)$ & $0.00035(11)$ & $0.00636(13)$ & $0.00011(12)$ \\
C11 & $0.0320(4)$ & $0.0439(4)$ & $0.0214(3)$ & $-0.0047(3)$ & $0.0130(3)$ & $0.0036(3)$ \\
O1 & $0.0285(10)$ & $0.0222(9)$ & $0.0181(9)$ & $0.0016(8)$ & $0.0076(8)$ & $-0.0014(7)$ \\
N1 & $0.0167(10)$ & $0.0209(11)$ & $0.0216(11)$ & $0.0035(8)$ & $0.0072(9)$ & $0.0015(8)$ \\
N2 & $0.0177(10)$ & $0.0202(10)$ & $0.0215(10)$ & $0.0012(8)$ & $0.0088(9)$ & $0.0001(8)$ \\
N3 & $0.0210(11)$ & $0.0203(11)$ & $0.0238(11)$ & $0.0001(9)$ & $0.0062(9)$ & $-0.0002(9)$ \\
C1 & $0.0183(12)$ & $0.0243(13)$ & $0.0183(12)$ & $0.0059(10)$ & $0.0054(10)$ & $0.0001(10)$ \\
C2 & $0.0230(12)$ & $0.0228(12)$ & $0.0200(12)$ & $0.0049(10)$ & $0.0036(10)$ & $-0.0025(10)$ \\
C3 & $0.0204(12)$ & $0.0315(14)$ & $0.0190(12)$ & $0.0063(11)$ & $0.0064(10)$ & $-0.0022(11)$ \\
C4 & $0.0198(12)$ & $0.0304(14)$ & $0.0222(13)$ & $0.0036(10)$ & $0.0092(10)$ & $0.0040(11)$ \\
C5 & $0.0180(12)$ & $0.0254(13)$ & $0.0241(13)$ & $0.0006(10)$ & $0.0078(10)$ & $-0.0024(11)$ \\
C6 & $0.0171(11)$ & $0.0261(13)$ & $0.0196(12)$ & $0.0047(10)$ & $0.0070(10)$ & $-0.0001(10)$ \\
C7 & $0.0188(12)$ & $0.0246(13)$ & $0.0211(12)$ & $0.0017(10)$ & $0.0085(10)$ & $0.0024(10)$ \\
C8 & $0.0160(11)$ & $0.0228(12)$ & $0.0197(12)$ & $0.0032(9)$ & $0.0055(10)$ & $0.0023(10)$ \\
C9 & $0.0195(12)$ & $0.0238(13)$ & $0.0276(13)$ & $-0.0001(10)$ & $0.0085(11)$ & $0.0055(11)$ \\
C10 & $0.0218(13)$ & $0.0217(12)$ & $0.0310(14)$ & $-0.0024(10)$ & $0.0049(11)$ & $-0.0047(11)$ \\
C11 & $0.0241(13)$ & $0.0267(13)$ & $0.0221(13)$ & $0.0019(11)$ & $0.0048(11)$ & $-0.0039(11)$ \\
C12 & $0.0252(13)$ & $0.0232(13)$ & $0.0206(12)$ & $0.0038(10)$ & $0.0077(11)$ & $0.0003(10)$ \\
C13 & $0.0167(11)$ & $0.0183(12)$ & $0.0209(12)$ & $0.0025(9)$ & $0.0062(10)$ & $0.0012(10)$ \\
C14 & $0.0189(12)$ & $0.0216(12)$ & $0.0203(12)$ & $0.0019(10)$ & $0.0080(10)$ & $-0.0009(10)$ \\
C15 & $0.0207(12)$ & $0.0182(12)$ & $0.0279(13)$ & $0.0029(10)$ & $0.0115(11)$ & $0.0015(10)$ \\
C16 & $0.0242(13)$ & $0.0246(13)$ & $0.0274(14)$ & $0.0022(11)$ & $0.0116(11)$ & $-0.0010(11)$ \\
C17 & $0.0302(14)$ & $0.0236(13)$ & $0.0291(14)$ & $0.0032(11)$ & $0.0158(12)$ & $0.0052(11)$ \\
C18 & $0.0273(13)$ & $0.0204(12)$ & $0.0364(15)$ & $0.0002(11)$ & $0.0160(12)$ & $0.0038(11)$ \\
C19 & $0.0216(12)$ & $0.0211(13)$ & $0.0330(15)$ & $-0.0002(10)$ & $0.0087(11)$ & $0.0007(11)$ \\
C20 & $0.0165(11)$ & $0.0193(12)$ & $0.0285(13)$ & $0.0055(9)$ & $0.0094(10)$ & $0.0044(10)$ \\
& & & & & & \\
\hline
\end{tabular}

Geometric parameters $\left(A,{ }^{\circ}\right)$

\begin{tabular}{llll}
\hline $\mathrm{Ni} 1-\mathrm{N} 3$ & $1.831(2)$ & $\mathrm{C} 8-\mathrm{C} 13$ & $1.416(3)$ \\
$\mathrm{N} 1-\mathrm{O} 1$ & $1.8617(18)$ & $\mathrm{C} 8-\mathrm{C} 9$ & $1.416(4)$ \\
$\mathrm{N} i 1-\mathrm{N} 1$ & $1.878(2)$ & $\mathrm{C} 9-\mathrm{C} 10$ & $1.382(4)$ \\
$\mathrm{N} 11-\mathrm{N} 2$ & $1.896(2)$ & $\mathrm{C} 9-\mathrm{H} 9$ & 0.9500 \\
$\mathrm{C} 11-\mathrm{C} 4$ & $1.748(3)$ & $\mathrm{C} 10-\mathrm{C} 11$ & $1.383(4)$ \\
$\mathrm{O} 1-\mathrm{C} 1$ & $1.324(3)$ & $\mathrm{C} 10-\mathrm{H} 10$ & 0.9500 \\
$\mathrm{~N} 1-\mathrm{C} 7$ & $1.293(3)$ & $\mathrm{C} 11-\mathrm{C} 12$ & $1.381(4)$ \\
$\mathrm{N} 1-\mathrm{C} 6$ & $1.430(3)$ & $\mathrm{C} 11-\mathrm{H} 11$ & 0.9500 \\
$\mathrm{~N} 2-\mathrm{C} 14$ & $1.333(3)$ & $\mathrm{C} 12-\mathrm{C} 13$ & $1.410(3)$ \\
$\mathrm{N} 2-\mathrm{C} 13$ & $1.424(3)$ & $\mathrm{C} 12-\mathrm{H} 12$ & 0.9500 \\
$\mathrm{~N} 3-\mathrm{C} 20$ & $1.329(3)$ & $\mathrm{C} 14-\mathrm{C} 15$ & $1.402(3)$
\end{tabular}




\begin{tabular}{|c|c|c|c|}
\hline $\mathrm{N} 3-\mathrm{H} 1$ & $0.836(18)$ & $\mathrm{C} 14-\mathrm{H} 14$ & 0.9500 \\
\hline $\mathrm{C} 1-\mathrm{C} 6$ & $1.403(4)$ & $\mathrm{C} 15-\mathrm{C} 16$ & $1.424(4)$ \\
\hline $\mathrm{C} 1-\mathrm{C} 2$ & $1.412(3)$ & $\mathrm{C} 15-\mathrm{C} 20$ & $1.432(4)$ \\
\hline $\mathrm{C} 2-\mathrm{C} 3$ & $1.381(4)$ & $\mathrm{C} 16-\mathrm{C} 17$ & $1.369(4)$ \\
\hline $\mathrm{C} 2-\mathrm{H} 2$ & 0.9500 & $\mathrm{C} 16-\mathrm{H} 16$ & 0.9500 \\
\hline $\mathrm{C} 3-\mathrm{C} 4$ & $1.395(4)$ & $\mathrm{C} 17-\mathrm{C} 18$ & $1.425(4)$ \\
\hline $\mathrm{C} 3-\mathrm{H} 3$ & 0.9500 & $\mathrm{C} 17-\mathrm{H} 17$ & 0.9500 \\
\hline $\mathrm{C} 4-\mathrm{C} 5$ & $1.382(4)$ & $\mathrm{C} 18-\mathrm{C} 19$ & $1.367(4)$ \\
\hline $\mathrm{C} 5-\mathrm{C} 6$ & $1.392(4)$ & $\mathrm{C} 18-\mathrm{H} 18$ & 0.9500 \\
\hline C5-H5 & 0.9500 & $\mathrm{C} 19-\mathrm{C} 20$ & $1.436(4)$ \\
\hline $\mathrm{C} 7-\mathrm{C} 8$ & $1.436(3)$ & C19-H19 & 0.9500 \\
\hline $\mathrm{C} 7-\mathrm{H} 7$ & 0.9500 & & \\
\hline $\mathrm{N} 3-\mathrm{Ni} 1-\mathrm{O} 1$ & $83.57(9)$ & $\mathrm{C} 13-\mathrm{C} 8-\mathrm{C} 7$ & $125.0(2)$ \\
\hline $\mathrm{N} 3-\mathrm{Ni1}-\mathrm{N} 1$ & $169.92(10)$ & $\mathrm{C} 9-\mathrm{C} 8-\mathrm{C} 7$ & $115.8(2)$ \\
\hline $\mathrm{O} 1-\mathrm{Ni} 1-\mathrm{N} 1$ & $86.35(9)$ & $\mathrm{C} 10-\mathrm{C} 9-\mathrm{C} 8$ & $121.7(2)$ \\
\hline $\mathrm{N} 3-\mathrm{Ni} 1-\mathrm{N} 2$ & $94.46(10)$ & $\mathrm{C} 10-\mathrm{C} 9-\mathrm{H} 9$ & 119.1 \\
\hline $\mathrm{O} 1-\mathrm{Ni} 1-\mathrm{N} 2$ & $176.53(9)$ & $\mathrm{C} 8-\mathrm{C} 9-\mathrm{H} 9$ & 119.1 \\
\hline $\mathrm{N} 1-\mathrm{Ni} 1-\mathrm{N} 2$ & $95.61(9)$ & $\mathrm{C} 9-\mathrm{C} 10-\mathrm{C} 11$ & $118.5(2)$ \\
\hline $\mathrm{C} 1-\mathrm{O} 1-\mathrm{Ni} 1$ & $112.57(16)$ & $\mathrm{C} 9-\mathrm{C} 10-\mathrm{H} 10$ & 120.8 \\
\hline $\mathrm{C} 7-\mathrm{N} 1-\mathrm{C} 6$ & $120.7(2)$ & $\mathrm{C} 11-\mathrm{C} 10-\mathrm{H} 10$ & 120.8 \\
\hline $\mathrm{C} 7-\mathrm{N} 1-\mathrm{Ni} 1$ & $128.02(18)$ & $\mathrm{C} 12-\mathrm{C} 11-\mathrm{C} 10$ & $121.4(3)$ \\
\hline $\mathrm{C} 6-\mathrm{N} 1-\mathrm{Ni} 1$ & $111.23(16)$ & $\mathrm{C} 12-\mathrm{C} 11-\mathrm{H} 11$ & 119.3 \\
\hline $\mathrm{C} 14-\mathrm{N} 2-\mathrm{C} 13$ & $114.6(2)$ & $\mathrm{C} 10-\mathrm{C} 11-\mathrm{H} 11$ & 119.3 \\
\hline $\mathrm{C} 14-\mathrm{N} 2-\mathrm{Ni} 1$ & $121.00(18)$ & $\mathrm{C} 11-\mathrm{C} 12-\mathrm{C} 13$ & $121.3(2)$ \\
\hline $\mathrm{C} 13-\mathrm{N} 2-\mathrm{Ni1}$ & $124.14(16)$ & $\mathrm{C} 11-\mathrm{C} 12-\mathrm{H} 12$ & 119.3 \\
\hline $\mathrm{C} 20-\mathrm{N} 3-\mathrm{Ni} 1$ & 131.89 (19) & $\mathrm{C} 13-\mathrm{C} 12-\mathrm{H} 12$ & 119.3 \\
\hline $\mathrm{C} 20-\mathrm{N} 3-\mathrm{H} 1$ & $122(2)$ & $\mathrm{C} 12-\mathrm{C} 13-\mathrm{C} 8$ & $117.5(2)$ \\
\hline $\mathrm{Ni} 1-\mathrm{N} 3-\mathrm{H} 1$ & $106(2)$ & $\mathrm{C} 12-\mathrm{C} 13-\mathrm{N} 2$ & $121.0(2)$ \\
\hline $\mathrm{O} 1-\mathrm{C} 1-\mathrm{C} 6$ & $118.0(2)$ & $\mathrm{C} 8-\mathrm{C} 13-\mathrm{N} 2$ & $121.5(2)$ \\
\hline $\mathrm{O} 1-\mathrm{C} 1-\mathrm{C} 2$ & $123.2(2)$ & $\mathrm{N} 2-\mathrm{C} 14-\mathrm{C} 15$ & $128.9(2)$ \\
\hline $\mathrm{C} 6-\mathrm{C} 1-\mathrm{C} 2$ & $118.7(2)$ & $\mathrm{N} 2-\mathrm{C} 14-\mathrm{H} 14$ & 115.6 \\
\hline $\mathrm{C} 3-\mathrm{C} 2-\mathrm{C} 1$ & 120.0 & $\mathrm{C} 15-\mathrm{C} 14-\mathrm{H} 14$ & 115.6 \\
\hline $\mathrm{C} 3-\mathrm{C} 2-\mathrm{H} 2$ & 120.0 & $\mathrm{C} 14-\mathrm{C} 15-\mathrm{C} 16$ & $118.3(2)$ \\
\hline $\mathrm{C} 1-\mathrm{C} 2-\mathrm{H} 2$ & 120.0 & $\mathrm{C} 14-\mathrm{C} 15-\mathrm{C} 20$ & $122.2(2)$ \\
\hline $\mathrm{C} 2-\mathrm{C} 3-\mathrm{C} 4$ & $119.7(2)$ & $\mathrm{C} 16-\mathrm{C} 15-\mathrm{C} 20$ & $119.5(2)$ \\
\hline $\mathrm{C} 2-\mathrm{C} 3-\mathrm{H} 3$ & 120.1 & $\mathrm{C} 17-\mathrm{C} 16-\mathrm{C} 15$ & $121.3(3)$ \\
\hline $\mathrm{C} 4-\mathrm{C} 3-\mathrm{H} 3$ & 120.1 & $\mathrm{C} 17-\mathrm{C} 16-\mathrm{H} 16$ & 119.3 \\
\hline $\mathrm{C} 5-\mathrm{C} 4-\mathrm{C} 3$ & $121.8(2)$ & $\mathrm{C} 15-\mathrm{C} 16-\mathrm{H} 16$ & 119.3 \\
\hline $\mathrm{C} 5-\mathrm{C} 4-\mathrm{Cl} 1$ & 119.0 & $\mathrm{C} 16-\mathrm{C} 17-\mathrm{C} 18$ & $119.1(3)$ \\
\hline $\mathrm{C} 3-\mathrm{C} 4-\mathrm{Cl} 1$ & $119.2(2)$ & $\mathrm{C} 16-\mathrm{C} 17-\mathrm{H} 17$ & 120.5 \\
\hline $\mathrm{C} 4-\mathrm{C} 5-\mathrm{C} 6$ & $118.3(2)$ & $\mathrm{C} 18-\mathrm{C} 17-\mathrm{H} 17$ & 120.5 \\
\hline $\mathrm{C} 4-\mathrm{C} 5-\mathrm{H} 5$ & 120.8 & $\mathrm{C} 19-\mathrm{C} 18-\mathrm{C} 17$ & $121.5(2)$ \\
\hline $\mathrm{C} 6-\mathrm{C} 5-\mathrm{H} 5$ & 120.8 & $\mathrm{C} 19-\mathrm{C} 18-\mathrm{H} 18$ & 119.3 \\
\hline $\mathrm{C} 5-\mathrm{C} 6-\mathrm{C} 1$ & $121.4(2)$ & $\mathrm{C} 17-\mathrm{C} 18-\mathrm{H} 18$ & 119.3 \\
\hline $\mathrm{C} 5-\mathrm{C} 6-\mathrm{N} 1$ & $126.9(2)$ & $\mathrm{C} 18-\mathrm{C} 19-\mathrm{C} 20$ & $120.6(3)$ \\
\hline $\mathrm{C} 1-\mathrm{C} 6-\mathrm{N} 1$ & $111.7(2)$ & $\mathrm{C} 18-\mathrm{C} 19-\mathrm{H} 19$ & 119.7 \\
\hline $\mathrm{N} 1-\mathrm{C} 7-\mathrm{C} 8$ & $123.8(2)$ & $\mathrm{C} 20-\mathrm{C} 19-\mathrm{H} 19$ & 119.7 \\
\hline
\end{tabular}




\begin{tabular}{|c|c|c|c|}
\hline $\mathrm{N} 1-\mathrm{C} 7-\mathrm{H} 7$ & 118.1 & $\mathrm{~N} 3-\mathrm{C} 20-\mathrm{C} 15$ & $119.2(2)$ \\
\hline $\mathrm{C} 8-\mathrm{C} 7-\mathrm{H} 7$ & 118.1 & $\mathrm{~N} 3-\mathrm{C} 20-\mathrm{C} 19$ & $122.9(2)$ \\
\hline $\mathrm{C} 13-\mathrm{C} 8-\mathrm{C} 9$ & $119.2(2)$ & $\mathrm{C} 15-\mathrm{C} 20-\mathrm{C} 19$ & $117.8(2)$ \\
\hline $\mathrm{N} 3-\mathrm{Ni} 1-\mathrm{O} 1-\mathrm{C} 1$ & $-176.59(18)$ & $\mathrm{Ni} 1-\mathrm{N} 1-\mathrm{C} 7-\mathrm{C} 8$ & $10.2(4)$ \\
\hline $\mathrm{N} 1-\mathrm{Ni1}-\mathrm{O} 1-\mathrm{C} 1$ & $3.42(17)$ & $\mathrm{N} 1-\mathrm{C} 7-\mathrm{C} 8-\mathrm{C} 13$ & $-4.8(4)$ \\
\hline $\mathrm{N} 3-\mathrm{Ni1}-\mathrm{N} 1-\mathrm{C} 7$ & $173.9(5)$ & $\mathrm{N} 1-\mathrm{C} 7-\mathrm{C} 8-\mathrm{C} 9$ & $172.8(2)$ \\
\hline $\mathrm{O} 1-\mathrm{Ni1}-\mathrm{N} 1-\mathrm{C} 7$ & $174.0(2)$ & $\mathrm{C} 13-\mathrm{C} 8-\mathrm{C} 9-\mathrm{C} 10$ & $2.6(4)$ \\
\hline $\mathrm{N} 2-\mathrm{Ni} 1-\mathrm{N} 1-\mathrm{C} 7$ & $-3.1(2)$ & $\mathrm{C} 7-\mathrm{C} 8-\mathrm{C} 9-\mathrm{C} 10$ & $-175.2(2)$ \\
\hline $\mathrm{N} 3-\mathrm{Ni1}-\mathrm{N} 1-\mathrm{C} 6$ & $-3.6(6)$ & $\mathrm{C} 8-\mathrm{C} 9-\mathrm{C} 10-\mathrm{C} 11$ & $2.7(4)$ \\
\hline $\mathrm{O} 1-\mathrm{Ni} 1-\mathrm{N} 1-\mathrm{C} 6$ & $-3.50(16)$ & $\mathrm{C} 9-\mathrm{C} 10-\mathrm{C} 11-\mathrm{C} 12$ & $-3.2(4)$ \\
\hline $\mathrm{N} 2-\mathrm{Ni} 1-\mathrm{N} 1-\mathrm{C} 6$ & $179.37(16)$ & $\mathrm{C} 10-\mathrm{C} 11-\mathrm{C} 12-\mathrm{C} 13$ & $-1.7(4)$ \\
\hline N3-Ni1-N2-C14 & $-15.0(2)$ & $\mathrm{C} 11-\mathrm{C} 12-\mathrm{C} 13-\mathrm{C} 8$ & $6.9(4)$ \\
\hline $\mathrm{N} 1-\mathrm{Ni1}-\mathrm{N} 2-\mathrm{C} 14$ & $164.51(19)$ & $\mathrm{C} 11-\mathrm{C} 12-\mathrm{C} 13-\mathrm{N} 2$ & $-173.9(2)$ \\
\hline $\mathrm{N} 3-\mathrm{Ni} 1-\mathrm{N} 2-\mathrm{C} 13$ & $170.91(19)$ & $\mathrm{C} 9-\mathrm{C} 8-\mathrm{C} 13-\mathrm{C} 12$ & $-7.2(3)$ \\
\hline $\mathrm{N} 1-\mathrm{Ni} 1-\mathrm{N} 2-\mathrm{C} 13$ & $-9.6(2)$ & $\mathrm{C} 7-\mathrm{C} 8-\mathrm{C} 13-\mathrm{C} 12$ & $170.3(2)$ \\
\hline $\mathrm{O} 1-\mathrm{Ni1}-\mathrm{N} 3-\mathrm{C} 20$ & $-171.4(2)$ & $\mathrm{C} 9-\mathrm{C} 8-\mathrm{C} 13-\mathrm{N} 2$ & $173.6(2)$ \\
\hline $\mathrm{N} 1-\mathrm{Ni1}-\mathrm{N} 3-\mathrm{C} 20$ & $-171.3(4)$ & $\mathrm{C} 7-\mathrm{C} 8-\mathrm{C} 13-\mathrm{N} 2$ & $-8.9(4)$ \\
\hline $\mathrm{N} 2-\mathrm{Ni} 1-\mathrm{N} 3-\mathrm{C} 20$ & $5.7(2)$ & $\mathrm{C} 14-\mathrm{N} 2-\mathrm{C} 13-\mathrm{C} 12$ & $22.3(3)$ \\
\hline $\mathrm{Ni} 1-\mathrm{O} 1-\mathrm{C} 1-\mathrm{C} 6$ & $-2.6(3)$ & $\mathrm{Ni1}-\mathrm{N} 2-\mathrm{C} 13-\mathrm{C} 12$ & $-163.25(19)$ \\
\hline $\mathrm{Ni} 1-\mathrm{O} 1-\mathrm{C} 1-\mathrm{C} 2$ & $177.86(19)$ & $\mathrm{C} 14-\mathrm{N} 2-\mathrm{C} 13-\mathrm{C} 8$ & $-158.5(2)$ \\
\hline $\mathrm{O} 1-\mathrm{C} 1-\mathrm{C} 2-\mathrm{C} 3$ & $-178.4(2)$ & $\mathrm{Ni} 1-\mathrm{N} 2-\mathrm{C} 13-\mathrm{C} 8$ & $15.9(3)$ \\
\hline $\mathrm{C} 6-\mathrm{C} 1-\mathrm{C} 2-\mathrm{C} 3$ & $2.1(4)$ & $\mathrm{C} 13-\mathrm{N} 2-\mathrm{C} 14-\mathrm{C} 15$ & $-169.2(2)$ \\
\hline $\mathrm{C} 1-\mathrm{C} 2-\mathrm{C} 3-\mathrm{C} 4$ & $0.3(4)$ & $\mathrm{Ni1}-\mathrm{N} 2-\mathrm{C} 14-\mathrm{C} 15$ & $16.1(4)$ \\
\hline $\mathrm{C} 2-\mathrm{C} 3-\mathrm{C} 4-\mathrm{C} 5$ & $-1.8(4)$ & $\mathrm{N} 2-\mathrm{C} 14-\mathrm{C} 15-\mathrm{C} 16$ & $175.5(2)$ \\
\hline $\mathrm{C} 2-\mathrm{C} 3-\mathrm{C} 4-\mathrm{Cl} 1$ & $177.5(2)$ & $\mathrm{N} 2-\mathrm{C} 14-\mathrm{C} 15-\mathrm{C} 20$ & $-2.7(4)$ \\
\hline $\mathrm{C} 3-\mathrm{C} 4-\mathrm{C} 5-\mathrm{C} 6$ & $0.9(4)$ & $\mathrm{C} 14-\mathrm{C} 15-\mathrm{C} 16-\mathrm{C} 17$ & $-174.8(2)$ \\
\hline $\mathrm{C} 11-\mathrm{C} 4-\mathrm{C} 5-\mathrm{C} 6$ & $-178.37(19)$ & $\mathrm{C} 20-\mathrm{C} 15-\mathrm{C} 16-\mathrm{C} 17$ & $3.5(4)$ \\
\hline $\mathrm{C} 4-\mathrm{C} 5-\mathrm{C} 6-\mathrm{C} 1$ & $1.5(4)$ & $\mathrm{C} 15-\mathrm{C} 16-\mathrm{C} 17-\mathrm{C} 18$ & $-0.4(4)$ \\
\hline $\mathrm{C} 4-\mathrm{C} 5-\mathrm{C} 6-\mathrm{N} 1$ & $178.9(2)$ & $\mathrm{C} 16-\mathrm{C} 17-\mathrm{C} 18-\mathrm{C} 19$ & $-1.3(4)$ \\
\hline $\mathrm{O} 1-\mathrm{C} 1-\mathrm{C} 6-\mathrm{C} 5$ & $177.4(2)$ & $\mathrm{C} 17-\mathrm{C} 18-\mathrm{C} 19-\mathrm{C} 20$ & $-0.2(4)$ \\
\hline $\mathrm{C} 2-\mathrm{C} 1-\mathrm{C} 6-\mathrm{C} 5$ & $-3.0(4)$ & $\mathrm{Ni1}-\mathrm{N} 3-\mathrm{C} 20-\mathrm{C} 15$ & $4.8(4)$ \\
\hline $\mathrm{O} 1-\mathrm{C} 1-\mathrm{C} 6-\mathrm{N} 1$ & $-0.2(3)$ & Ni1-N3-C20-C19 & $-177.35(19)$ \\
\hline $\mathrm{C} 2-\mathrm{C} 1-\mathrm{C} 6-\mathrm{N} 1$ & $179.3(2)$ & $\mathrm{C} 14-\mathrm{C} 15-\mathrm{C} 20-\mathrm{N} 3$ & $-8.6(4)$ \\
\hline $\mathrm{C} 7-\mathrm{N} 1-\mathrm{C} 6-\mathrm{C} 5$ & $7.7(4)$ & $\mathrm{C} 16-\mathrm{C} 15-\mathrm{C} 20-\mathrm{N} 3$ & $173.2(2)$ \\
\hline $\mathrm{Ni} 1-\mathrm{N} 1-\mathrm{C} 6-\mathrm{C} 5$ & $-174.6(2)$ & $\mathrm{C} 14-\mathrm{C} 15-\mathrm{C} 20-\mathrm{C} 19$ & $173.4(2)$ \\
\hline $\mathrm{C} 7-\mathrm{N} 1-\mathrm{C} 6-\mathrm{C} 1$ & $-174.8(2)$ & $\mathrm{C} 16-\mathrm{C} 15-\mathrm{C} 20-\mathrm{C} 19$ & $-4.8(3)$ \\
\hline $\mathrm{Ni} 1-\mathrm{N} 1-\mathrm{C} 6-\mathrm{C} 1$ & $2.9(3)$ & $\mathrm{C} 18-\mathrm{C} 19-\mathrm{C} 20-\mathrm{N} 3$ & $-174.7(2)$ \\
\hline $\mathrm{C} 6-\mathrm{N} 1-\mathrm{C} 7-\mathrm{C} 8$ & $-172.5(2)$ & $\mathrm{C} 18-\mathrm{C} 19-\mathrm{C} 20-\mathrm{C} 15$ & $3.2(4)$ \\
\hline
\end{tabular}

Hydrogen-bond geometry $\left(A,{ }^{\circ}\right)$

\begin{tabular}{lllll}
\hline$D-\mathrm{H} \cdots A$ & $D-\mathrm{H}$ & $\mathrm{H} \cdots A$ & $D \cdots A$ & $D-\mathrm{H} \cdots A$ \\
\hline $\mathrm{C} 12-\mathrm{H} 12 \cdots \mathrm{C} 11^{\mathrm{i}}$ & 0.95 & 2.76 & $3.540(3)$ & 140 \\
$\mathrm{C} 10-\mathrm{H} 10 \cdots \mathrm{C} 20^{\mathrm{ii}}$ & 0.95 & 2.80 & $3.626(4)$ & 146 \\
\hline
\end{tabular}

Symmetry codes: (i) $x, y, z-1$; (ii) $-x, y-1 / 2,-z+1 / 2$. 